\title{
Accurate WiFi Based Localization for Smartphones Using Peer Assistance
}

\author{
Hongbo Liu, Member, IEEE, Jie Yang, Member, IEEE, Simon Sidhom, Yan Wang, Student Member, IEEE, \\ Yingying Chen, Senior Member, IEEE, and Fan Ye
}

\begin{abstract}
Highly accurate indoor localization of smartphones is critical to enable novel location based features for users and businesses. In this paper, we first conduct an empirical investigation of the suitability of WiFi localization for this purpose. We find that although reasonable accuracy can be achieved, significant errors (e.g., $6 \sim 8 \mathrm{~m}$ ) always exist. The root cause is the existence of distinct locations with similar signatures, which is a fundamental limit of pure WiFi-based methods. Inspired by high densities of smartphones in public spaces, we propose a peer assisted localization approach to eliminate such large errors. It obtains accurate acoustic ranging estimates among peer phones, then maps their locations jointly against WiFi signature map subjecting to ranging constraints. We devise techniques for fast acoustic ranging among multiple phones and build a prototype. Experiments show that it can reduce the maximum and 80 -percentile errors to as small as $2 m$ and $1 m$, in time no longer than the original WiFi scanning, with negligible impact on battery lifetime.
\end{abstract}

Index Terms-Smartphone, peer assisted localization, WiFi fingerprint localization

\section{INTRODUCTION}

$I^{s}$ NDOOR localization is a critical enabler for location based smartphone applications. In many environments (e.g., airport terminals, railway stations and shopping malls), the location helps users access navigation, merchandise and promotion information; businesses need it to understand the patterns of customer visit and stay, such as the popularity of different sections in a store, or the spatial-temporal distribution of passenger flows.

Accurate indoor localization on smartphones, however, remains elusive. Although there have been some recent commercial offerings such as Google Maps 6.0 and Shopkick [1], they either have errors up to 10 meters [11], or only locate at the granularity of stores. There has been a plethora of academic work on indoor localization. Those achieving high accuracy usually require special hardware not readily available on smartphones [30], [31], [35], or infrastructure expensive to deploy [7], [21]. WiFi-based localization leverages prevalent wireless access points, thus

- H. Liu is with the Department of Computer Information and Graphics Technology, IUPUI, Indianapolis, IN 46222 USA.

E-mail: hl45@iupui.edu.

- Y. Wang and Y. Chen are with the Department of Electrical and Computer Engineering, Stevens Institute of Technology, Hoboken, NJ 07030 USA. E-mail: \{ywang10, yingying.chen\}@stevens.edu.

- J. Yang is with the Department of Computer Science, Florida State University, Tallahassee, FL 32306 USA.

E-mail: jie.yang@cs.fsu.edu.

- S. Sidhom is with the Department of Computer Science, Stevens Institute of Technology, Hoboken, NJ 07030 USA. E-mail: ssidhom@stevens.edu.

- F. Ye is with the Center for Energy-efficient Computing and Applications, EECS School, Peking University, Beijing 10087, China.

E-mail: yefan@pku.edu.cn.

Manuscript received 14 Jan. 2013; revised 14 July 2013; accepted 10 Oct. 2013. Date of publication 24 Oct. 2013; date of current version 26 Aug. 2014. For information on obtaining reprints of this article, please send e-mail to: reprints@ieee.org, and reference the Digital Object Identifier below.

Digital Object Identifier 10.1109/TMC.2013.140 avoiding such drawbacks. However, most WiFi-based localization [3], [13], [14], [16], [38] have been largely based on laptops with quite different antenna forms (e.g., antenna polarization) and possibly radio characteristics (e.g., multiple channels and power levels), whereas recent work on smartphone indoor localization [2], [9], [19] achieves room or floor level accuracies and Liu et al. [26] also proposes a MCMC based approach with mean error around 4 meters using a pocket-placed smartphone. The feasibility of leveraging the most prevalent WiFi infrastructure for high accuracy localization on smartphones is still an open question.

In this paper, we first conduct a set of experiments in two different environments (i.e., office and gym) to empirically study the impact of various factors on the accuracy of WiFi localization on smartphones. We find that although reasonable accuracy (e.g., $3 \sim 4 \mathrm{~m}$ ) can be achieved, there always exist large errors (e.g., $6 \sim 8 \mathrm{~m}$ ) unacceptable for many scenarios in both environments. Similar or much larger errors (e.g., $>15 \mathrm{~m}$ ) have been reported in previous studies [3], [38]. One work [8] found that high accuracy (e.g., sub-meter median and $2 \mathrm{~m}$ maximum) is possible but only under hundreds of APs, infeasible in practical settings. Such errors may cause a passenger to make a wrong turn leading to a different train platform, or a store to erroneously stock up for a section with much less real customer interests. Our investigation on these large errors reveals the insight that they are caused by possibly faraway locations with similar WiFi signatures, an intrinsic phenomenon of the radio signal propagation and fundamental limit of WiFi methods.

On the other hand, we observe that smartphones are gradually woven into our social life and usually a high density of them exist in public spaces. The relative positions of nearby peer devices could be used as physical constraints on the possible location of a smartphone. Inspired by 


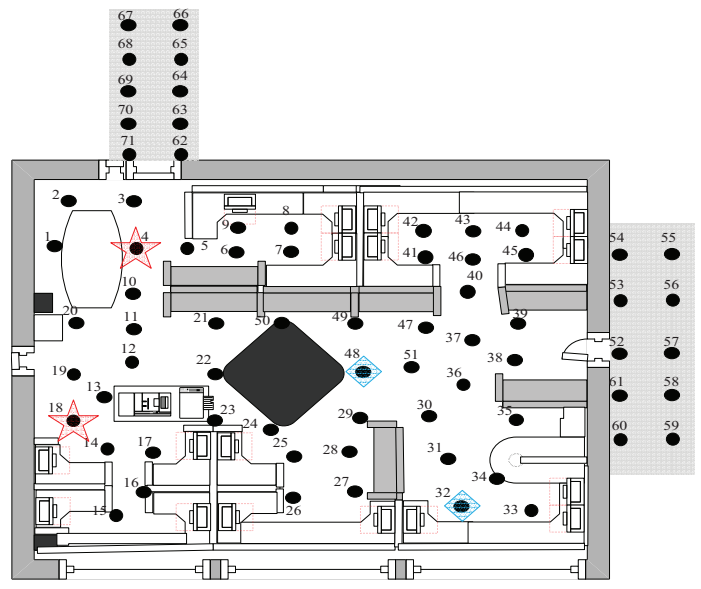

(a)

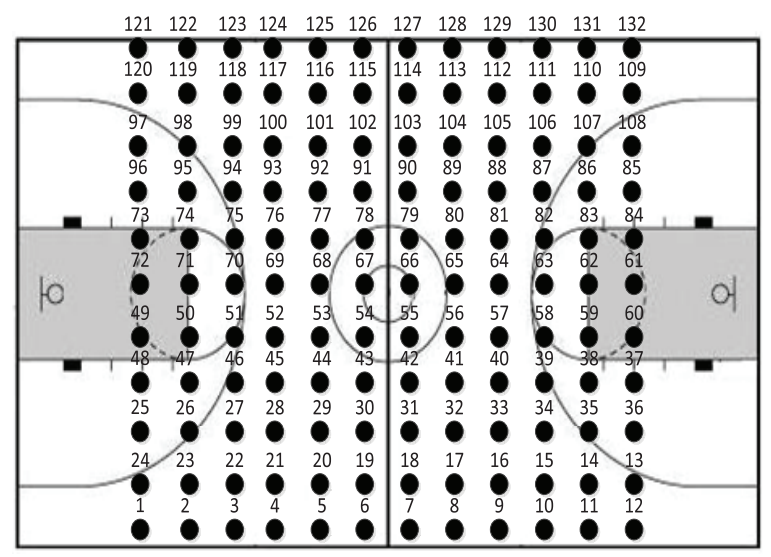

(b)

Fig. 1. Each dot in the floor map represents a location where the WiFi signal strength fingerprint is measured. (a) Office environment. (b) Gym environment.

this observation, we set out to study how to exploit the unique physical constraints among smartphones to reduce large errors and push the limit of WiFi based approaches. We propose a peer-phone assisted localization approach that leverages the acoustic ranging between peers, without requiring special hardware yet producing highly accurate location estimates.

In particular, the peer-phone assisted localization can be carried out concurrently with WiFi localization or when a smartphone has obtained a rough location from WiFi but needs further improvements. The targeting smartphone exchanges sound signals with nearby peer devices. A virtual synchronization scheme is proposed to avoid the interference of sound signals among multiple smartphones. A server collects acoustic ranging estimates and constructs a graph of the relative positions among peers. It applies a localization algorithm that maps the vertices of the graph against the WiFi signature database to locate all peers jointly. Experiments using data from various environments, including the airport, train station, and shopping mall, have shown that our approach can reduce 80 percentile error to about $1 \mathrm{~m}$, and limit the maximum error to about $2 \mathrm{~m}$, demonstrating the feasibility of WiFi for high accuracy localization.

Specifically, we make the following contributions:

- We discover the root cause of large errors as the existence of faraway locations sharing similar radio signatures, which is due to the intrinsic dynamic propagation of the radio signal, thus presenting a fundamental limit of WiFi methods.

- We propose a peer-phone assisted localization approach utilizing minimum auxiliary COTS sound hardware for reducing large errors and push the limit of WiFi approaches. We devise a peer-assisted localization algorithm that leverages acoustic ranging and locates peer phones jointly for greatly improved accuracy. We identify the frequencies, sound signal design, detection and emission scheduling methods appropriate for fast ranging among multiple peers that are unobtrusive, robust to noise and have minimum impact on users' regular activities.

- We prototype our system and carry out real world experiments. The results demonstrate that our approach greatly reduces the maximum error from $6-8 \mathrm{~m}$ to $2 \mathrm{~m}$, and limit 80 percentile error to $1 \mathrm{~m}$, which were shown empirically possible but only under hundreds of APs [8]. The assistance finishes in time no longer than a few seconds of WiFi scanning, and poses negligible impact on battery lifetime.

The rest of this paper is organized as follows. In Section 2, we perform a systematic evaluation on WiFi localization on smartphones and report our findings. Section 3 introduces our system design and present the detailed peer assisted localization algorithm. We study the frequencies, sound detection methods for fast concurrent ranging among multiple peer phones in Section 4. We describe the system implementation and report evaluation results in Section 5. We discuss related issues and survey related work in Sections 6 and 7. Finally, Section 8 concludes the paper.

\section{Performance of Using Wifi Alone for SMARTPHONE LOCALIZATION}

To understand the practical performance of smartphone localization using $\mathrm{WiFi}$, we first conduct a systematic study on the impact of various factors (e.g., orientation, holding position, time of the day and number of samples) in two different environments, an office environment with furniture and wall dividers and a gym area with open space, as shown in Fig. 1. In both environments, we find that reasonable accuracy can be achieved in many cases (e.g., $\sim 4 m$ ). However, large errors (e.g., beyond $6 \sim 8 m$ ) always exist. Further investigation reveals two root causes: static environmental effects, and dynamic obstacles or interferences, both of which pose fundamental limits on WiFi localization accuracy. 


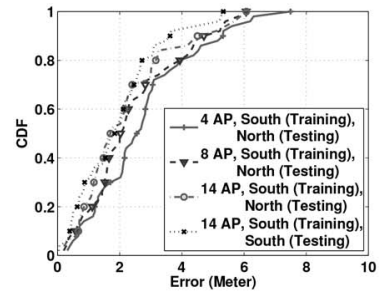

(a)

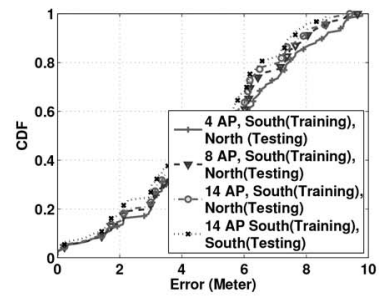

(e)

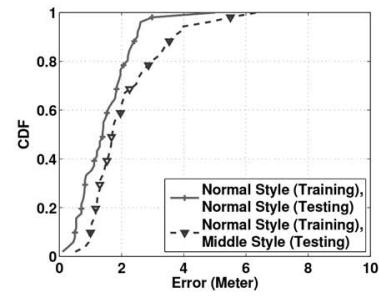

(b)

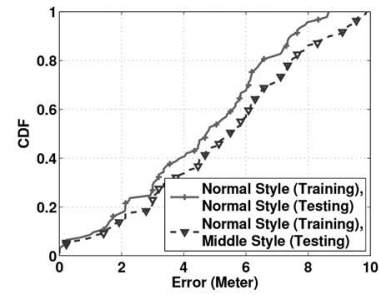

(f)

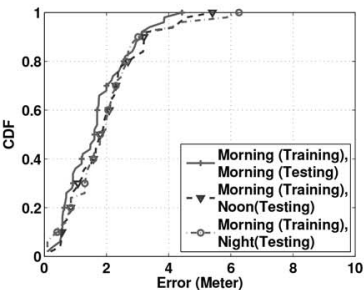

(c)

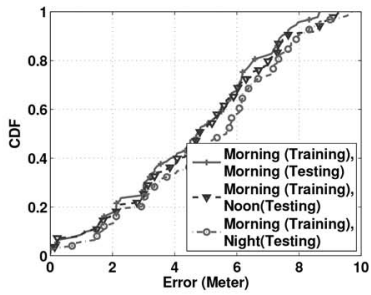

(g)

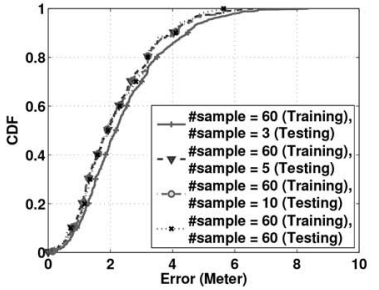

(d)

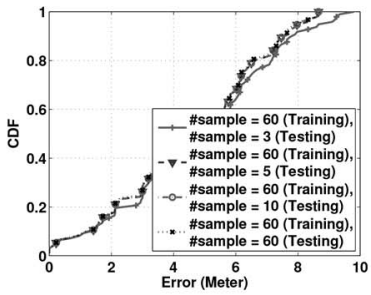

(h)

Fig. 2. WiFi localization error in office vs. gym under orientation, holding style, time of day and number of samples. (a) Orientation, office. (b) Holding style, office. (c) Time of day, office. (d) Number of samples, office. (e) Orientation, gym. (f) Holding style, gym. (g) Time of day, gym. (h) Number of samples, gym.

\subsection{Methodology}

Fingerprint Based Localization. Fingerprint based method was pioneered by Bahl et al. [4] and is the most popular WiFi localization approach. It first measures the "fingerprint", the WiFi signal strengths from various access points (APs) at a number of known locations and stores them as training data. A device samples the signal strengths from various APs to obtain testing data. Then an algorithm finds the "closest fingerprints" in the training data to the sample, using Euclidean distance in the signal space where each dimension is for a different AP. A location estimation is given based on the locations of the closest fingerprints (e.g., the centroid of a few "closest" fingerprint locations). Recent work [9], [10] showed that the training data could be constructed without extensive site survey, making this approach even more attractive. In the test, we build a fine-grained signature map as training data, using interpolation between locations with actual measurements.

Experimental Setup. Fig. 1 depicts the floor plan of our experimental sites, where the office is located at the second floor of Burchard building at Stevens Institute of Technology with the floor size of $12 \mathrm{~m} \times 11 \mathrm{~m}$ and the gym is an indoor basketball court with the size of $18 \mathrm{~m} \times 16 \mathrm{~m}$ located at the top floor of Walker gym at Stevens. The office is a typical indoor environment with hallways, office wall dividers and furniture, such as desks, shelves and chairs, whereas the gym is an open area with only two basket pole on the floor. We collect the WiFi signal strengths at 71 and 132 positions in office and gym, respectively. These locations are shown as small dots in Fig. 1 and the distance between two adjacent locations is around $1.5 \mathrm{~m}$ in both environments. In both environments, we choose 14 APs with large coverage in our localization process. At each location, the phone can observe the signals from 8-9 APs on average and we collect 60 Receive Signal Strength (RSS) readings for each observed AP. We repeat the above process by varying 4 different factors, orientation, holding position, time of the day and number of samples (shown in
Table 1), to understand how they impact the localization performance. For each test, the default parameters are south for orientation, normal style for holding position, 60 samples and morning time.

\subsection{Impact of Various Factors}

Fig. 2 presents the cumulative distribution function (CDF) of the localization error under various factors in both the office and gym environments.

We first examine how the orientation affects the RSS readings due to the blocking and reflection of radio signals by the human body. In outdoor cases, 10dB RSS difference for certain APs was observed on smartphones [40]. In our indoor environment, we recorded an average of $4 \mathrm{~dB}$ difference. We believe this reduced variation is due to stronger multipath effects of indoor environments, thus the lack of direct line of sight does not attenuate the signal as much. In particular, as shown in Fig. 2(a) and (e), when a mismatched training data set is used (e.g., south-facing training set for north-facing user), long tails of CDF curves exhibit large errors ranging from $6 m$ to $8 m$ in the office and $8 m$ to $10 m$ in the gym. Even when the matching training set is applied, errors beyond $5 \mathrm{~m}$ in the office and $8 \mathrm{~m}$ in the gym still exist.

Due to the small size, how the user's hand holds the phone can affect the received radio signal as well. We tried two holding positions: bottom and middle. Fig. 2(b) and (f) show that using mismatched training and testing data (e.g., bottom-holding as training to localize middle-holding phones) can lead to large error beyond $6 m$ in the office

TABLE 1

Factors Under Study

\begin{tabular}{|l|l|l|}
\hline \multicolumn{1}{|c|}{ Factors } & \multicolumn{1}{c|}{ Description } & \multicolumn{1}{c|}{ Value } \\
\hline \hline Orientation & $\begin{array}{l}\text { The direction to which the user is } \\
\text { facing }\end{array}$ & $\begin{array}{l}\text { West, North, } \\
\text { East, South }\end{array}$ \\
\hline Holding Position & The way the user holds the phone & $\begin{array}{l}\text { Holding bottom, } \\
\text { Holding middle }\end{array}$ \\
\hline $\begin{array}{l}\text { Number of } \\
\text { Samples }\end{array}$ & $\begin{array}{l}\text { The number of samples taken at } \\
\text { each location }\end{array}$ & $3,5,10,60$ \\
\hline Time of the Day & $\begin{array}{l}\text { Different times that we conduct the } \\
\text { measurements }\end{array}$ & $\begin{array}{l}\text { Morning, Noon, } \\
\text { Night }\end{array}$ \\
\hline
\end{tabular}


and $8 m$ in the gym. In addition, the localization results are evaluated under three different times of the day representing morning, noon and evening in Fig. 2(c) and (g). We again observe long tails beyond 5 meters in the office and 8 meters in the gym when mismatched training and testing data are used.

Finally, more samples lead to more reliable measurements as the input for localization, but at the cost of higher energy and latency overhead. To understand the accuracyoverhead tradeoff, we depict the localization error when varying the number of samples from 3 to 60 in Fig. 2(d) and (h) under a training set of 60 samples per location. We find that using more than 5 samples does not significantly improve 90-percentile accuracy. We thus choose 5 samples in our WiFi localization study throughout the paper.

We find that large errors in both environments are always over 6 meters. Results in the gym environment are about 2 meters worse than those in the office environment for both maximum and median errors. This is because there are few obstacles in an empty indoor environment (i.e., gym), and thus the wireless signals in the gym experience less attenuation than those in the office environment. In particular, we find that the average distance between the signatures of two nearby locations in the signal space is approximately $1.4 \mathrm{~dB} \backslash A P$ in the gym, whereas it is around $2.1 \mathrm{~dB} \backslash A P$ in the office. Consequently, it is hard to discriminate the signatures between nearby points in gym when performing fingerprint localization.

\subsection{Root Cause of Large Errors}

We make one critical observation from the above investigation: although reasonable accuracy can be achieved in many cases, large errors $6 m$ or more always exist in both environments. In many applications these large errors can cause problems, such as giving the user incorrect navigation instructions, or wrong statistics about the visits of customers to different sections inside a store.

After a close examination of those large errors, we find the root cause. In essence, two physically distant locations happen to share similar WiFi signal strength measurements, thus a testing sample is erroneously localized to a physically faraway location with short Euclidean distance in the signal space. This can be classified into two cases: (1) permanent environmental settings such as walls, furniture placement, which affect radio signal propagation and create persistent similar signal reception; and (2) transient factors or measurement mismatch between training and testing data. Such transient variation in RSS reception is due to dynamic changes in the environment, such as a nearby moving object or wireless interference from other electronic devices, while the mismatch can be in orientation, holding style, time of the day or number of samples.

We illustrate case 1 by three locations in Fig. 1: 18 and 13 are close to each other whereas 4 is farther at the other side of the room (marked as red stars). However, during testing we find that locations 18 and 4 share similar WiFi fingerprints. The distance between their fingerprints in the signal space is only $1.98 \mathrm{~dB} / \mathrm{AP}$, whereas the fingerprint at closeby location 13 has a distance of $2.44 \mathrm{~dB} / \mathrm{AP}$ to that of location 18. In this case, the office wall dividers cause the large localization error beyond $6 \mathrm{~m}$ at location 18 .
The example for case 2 are location 32, 34 and 48 (marked as blue squares in Fig. 1). Locations 32 and 34 are close to each other. However, we find that the WiFi fingerprint at location 32 becomes similar to that at location 48 at night when less people are around. Thus when testing at location 32 using the training data collected at night, location 32 will be matched to 48 , instead of 34 , resulting in large errors of over $6 \mathrm{~m}$. Through our study, we find that the percentage of large errors resulted from Case 1 is $60 \%$ to $70 \%$ in both environments while that from Case 2 accounts for the rest. Both cases are caused by irregular multipath reflections, an intrinsic character of radio signals. They present fundamental limits for WiFi localization to achieve high accuracy.

\section{Peer Assisted localization}

From the previous investigation, WiFi as-is is not a suitable candidate for high accuracy localization due to large errors. However, is it possible to address this fundamental limit without the need for additional hardware or infrastructure? Our answer is yes: by exploiting acoustic ranging, a phone can use nearby peer phones as reference points and obtain its relative positions to them. This imposes unique physical constraints on the possible location of the phone, thus reducing the uncertainty and improving the accuracy.

Such an idea is motivated by two observations. First, in many public indoor environments (e.g., airport terminals, railway stations, shopping malls and museums), there are usually a high density of users, thus smartphones. Each neighboring peer has a unique physical location for restraining the location uncertainty of a smartphone. Second, a number of research work [29], [37] has shown that highly accurate relative ranging can be achieved within a car (passenger vs. driver side) or between a pair of mobile devices (at centimeter accuracy) by using sound signals.

\subsection{Design Goals and Challenges}

The above concept may sound quite simple. However, building such a peer-assisted localization system involves a number of great challenges in both the design and implementation:

- Peer assisted localization algorithm. How to utilize the physical constrains imposed by the neighborhood peers to reduce the large errors incurred from WiFi localization? Given only the relative distances among peers and their location estimates are available in real scenarios, exactly what is the algorithm?

- Concurrent acoustic ranging of multiple phones. Previous work on acoustic position estimation was for one or two devices only. When there are multiple devices and they all do acoustic ranging, how can we tell which distance measure is for which pair? How to design and detect the sound signal, so that the system is robust to noises in different environments?

- $\quad$ Ease of use. The peer assistance process should complete in short time; otherwise users may have moved to different locations. The sound emitted should not annoy or distract users from their regular activities. 


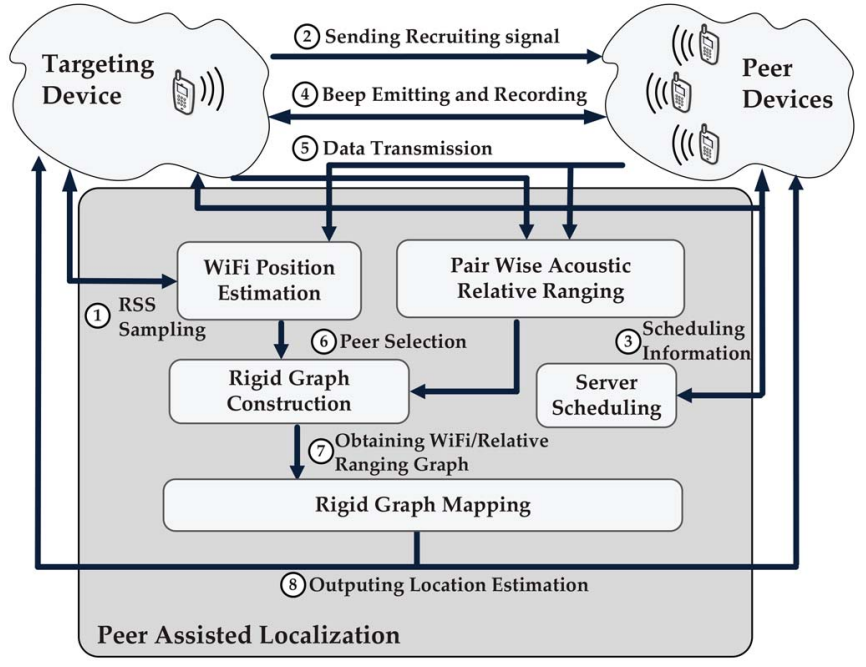

Fig. 3. Workflow of our system.

In the rest of this section, we will present an overview of the system, then present the details of the peer assisted localization algorithm. Section 4 addresses challenges in concurrent acoustic ranging and ease of use.

\subsection{System and Algorithm Overview}

Our system works as follows (shown in Fig. 3): when a target phone needs to further improve accuracy or desires high accuracy upfront, it broadcasts a special audio signal to "recruit" nearby peers. Those receiving the recruiting signal send their identifiers to a server. The server comes up with a schedule about which phone should emit a beep signal at which time slot. The phones involved, both the target and peers, emit the beeps accordingly. They also record the beeps from others and send the files back to the server. All peers also conduct WiFi sampling and send the measurements to the server. The server determines the locations of peers from WiFi samples, and distances among them from acoustic ranging based on the recorded sound files. Finally the server computes the new location estimate of the target using our peer assisted localization algorithm, and sends back the result to the target.

The intuition underlying the peer assisted localization algorithm is to construct a graph based on the relative distances among devices, then "superimpose" the graph onto the signature map based on the initial WiFi location estimates. The algorithm "rotates" and translationally "moves" the graph against the signature map, such that the vertices are placed "closest" to the true locations, as measured by certain metric, where the vertices are placed become the new location estimates.

In the algorithm, we use as the metric the sum of RSS Euclidean distances between the WiFi samples of each device and the WiFi signature of where its graph vertex is placed. Such a metric quantifies the aggregate "closeness" between WiFi samples and new estimated locations of all devices. We use the example in Fig. 4 to illustrate the intuition. Initially, each phone has a location estimate

1. "Translationally" means moving the graph without rotation.
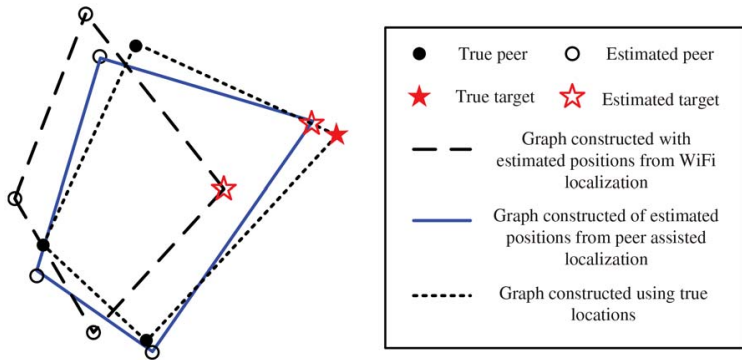

Fig. 4. Illustration of using physical constraints to perform peer assisted localization.

(vertices in the dashed-line graph), e.g., from WiFi localization. Because the acoustic ranging is highly accurate to identify the relative distances between peers (in terms of centimeters), the shape of the solid-line graph is quite close to that of the ground truth (the dotted-line graph). Such additional relative distance constraints "force" the new location estimate of the target to move closer to its real location, thus reducing large localization errors and achieving higher accuracy.

\subsection{Peer Assisted Localization Algorithm}

Our peer assisted algorithm comprises of two main parts: (a) graph orientation estimation: estimating the range of graph orientation angle $\phi^{2}$ by combining the acoustic ranging information and initial WiFi localization results. This is important because we find that its performance is sensitive to the orientation (shown in Section 5.5). (b) new location estimation: search for the optimal combination of graph orientation angle and translational position, such that the RSS distance summation is minimized.

The algorithm takes two inputs: 1) $\left\{p_{i}\right\}, i=0, \ldots, M$ : initial WiFi location estimations of the target phone $\left(p_{0}\right)$ and its peers $\left.\left(p_{1}, \ldots, p_{M}\right) ; 2\right)\left\{l_{i, j}\right\}, i, j=0, \ldots, M$ : pairwise acoustic ranging measurements between device $i$ and $j$. The algorithm produces $\left\{q_{i}\right\}$, the new location estimate of each involved device $i$. In the following, the first 3 steps estimate the orientation range, and the last two find the new locations.

Step 1: Compute edge directions from acoustic ranging. The server constructs a graph $G^{\prime}$ with the pairwise ranging measurements, $l_{i, j}, i, j \in\{0, \ldots, M\}$. It rotates $G^{\prime}$ such that the direction of its longest edge is parallel to the $X$ axis. Then it calculates the direction vector $v_{l}^{\prime}=\frac{p_{i}^{\prime}-p_{j}^{\prime}}{\left|p_{i}^{\prime}-p_{j}^{\prime}\right|},(i<j)$ for the $L$ (e.g., 3$)^{3}$ longest edges, where $p_{i}^{\prime}, p_{j}^{\prime}$ are the locations of the two vertices of an edge.

Step 2: Compute edge directions from initial WiFi localization. The server also computes the direction vectors for the same $L$ edges using initial WiFi locations: $v_{l}=\frac{p_{i}-p_{j}}{\left|p_{i}-p_{j}\right|}$, $(i<j)$.

2. We define $\phi$ as the angle between the $X$ axis and the direction of the graph's longest edge, from the vertex of the smaller ID to the other.

3. When choosing $L$ longest edges, we experimented various values (e.g., 2, 3 and 4 ) and found different values of $L$ have little impact on the final localization results. We used the value of 3 as an example in our evaluation. 


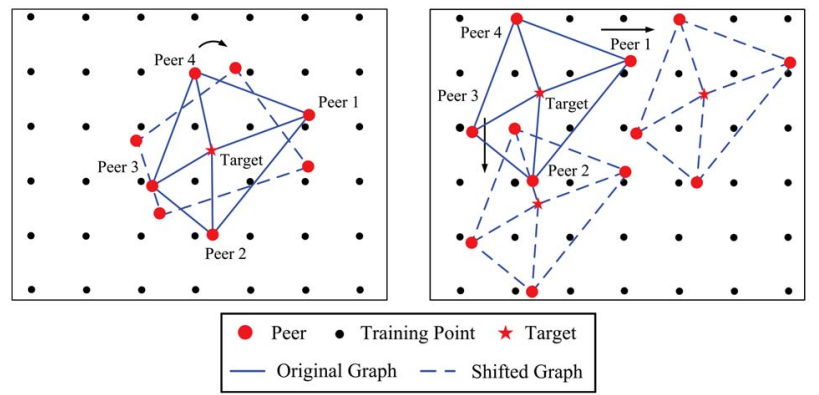

(a)

(b)

Fig. 5. Translational movements or rotation of a graph preserve the distance constraints between vertices. (a) Rotation. (b) Translational movements.

Step 3: Graph orientation estimation. The server rotates $G^{\prime}$ to find the optimal graph orientation $\Phi$ that maximizes the inner product summation between $\left\{v_{l}\right\}$ and $\left\{v_{l}^{\prime}\right\}$ :

$$
\Phi=\operatorname{argmax} \sum_{l=1}^{L} v_{l}^{\prime} v_{l}^{T} .
$$

The inner product is a monotonically decreasing function of the angle difference between two vectors. Thus Equation 1 minimizes the angle differences of $L$ edges obtained from acoustic ranging and initial WiFi localization.

Due to ranging errors, the real orientation may differ from $\Phi$. To ensure that the true orientation is covered, the search of $\left\{q_{i}\right\}$ is conducted in an orientation range of [ $\Phi-$ $\Delta \Phi, \Phi+\Delta \Phi]$, where $\Delta \Phi=20^{\circ}$ from our empirical study in Section 5.5. We choose the $L$ longest edges because the longer an edge, the less the impact on its direction caused by the same ranging error.

Step 4: Set the search scopes. Based on the initial WiFi position estimation $p_{i}$, the server tries to superimpose $G^{\prime}$ onto the WiFi signature map, such that each vertex $i$ except the target is restricted inside a small circle $A_{i}$ centered at $p_{i}$ with radius $r_{i}$ during the searching process in following step. We set $r_{i}$ at $2 m$ based on empirical study (see Section 5.1).

Step 5: Joint location estimation. Finally, with a small movement step of $\alpha$ meters and rotation step of $\beta$ degrees (set to $0.1 \mathrm{~m}$ and $2^{\circ}$ based on empirical study), the server searches for the optimal location and orientation combination to superimpose the graph against the fingerprint map using the following objective function, where the moving and rotating operations are illustrated in Fig. 5(a) and (b) respectively:

$$
\underset{\left\{q_{i}\right\}, q_{i} \in S}{\arg \min } \sum_{i=0}^{M}\left[f\left(q_{i}\right)-f\left(p_{i}\right)\right]\left[f\left(q_{i}\right)-f\left(p_{i}\right)\right]^{T},
$$

where $f(x)=\left[\begin{array}{lll}R S S_{x}^{1} & R S S_{x}^{2} \cdots R S S_{x}^{k}\end{array}\right]$ is the WiFi fingerprint or measurement at location $x$ (i.e., the RSS measurements of $k$ detected access points).

Equation 2 produces the final results by finding a set of locations $\left\{q_{i}\right\}, i=0,1, \ldots, M$ from the $\mathrm{WiFi}$ fingerprint database $S$, so as to minimize the summation of RSS distances, each of which is between device $i$ 's WiFi measurement $f\left(p_{i}\right)$ and the signature $f\left(q_{i}\right)$ at its new location estimation $q_{i}$.

One comment we want to make is that the distances between some pairs of vertices may not determine the shape of a graph uniquely. For example, a square is flexible since its vertices can rotate against each other and form a family of rhombi while preserving the edge lengths, whereas the shape of a triangle is "rigid" (i.e., uniquely determined) given the lengths of the three edges. The rigid graph theory [18] describes under what conditions a graph is rigid: A complete graph with a known distance between any two vertices is rigid. The concurrent ranging among multiple devices and the resulting pairwise distances give a complete, thus rigid graph. 4

Extensive evaluation shows that our algorithm greatly improves the localization accuracy of the target phone (details in Section 5). The improvement is limited only under some rare cases (e.g., when peers are aligned almost on a straight line, or clustered together and located far away from the target), which we discuss in Section 5.4.1.

\section{Acoustic Relative Ranging}

Our peer-assisted approach requires acoustic ranging among multiple phones. This raises two new issues: First, how to ensure that the signals from different peer phones do not interfere. Sound signals do not carry a MAC address so there is no way to tell which phone emitted which sound. Second, the whole process has to complete in short time. Otherwise users may have moved, causing inaccurate ranging results. In this section, we describe our signal design, detection, and scheduling techniques that satisfy the requirements of concurrent multi-peer ranging.

\subsection{Beep Signal Design and Detection}

\subsubsection{Acoustic Ranging Principle}

In principle, ranging can be done by Time-of-Arrival (TOA) method that estimates the sound travel time from one device to another. The difficulty is in uncertainties: both the emitting and detecting have variable delays difficult to measure. The lack of clock synchronization between devices further adds to the problem. To address these issues, we have each involved peer device emit signals and the uncertainties will cancel out each other in calculation, similar to Beepbeep [29]. By doing so, only delay measurements from the same device are needed, thus circumventing the above issues.

\subsubsection{Beep Design}

We need to design the acoustic ranging signal carefully. The signal should be robust to various background noise (e.g., human conversations, PA announcements and music) that make it hard to detect. It should have minimal disturbance to people's normal activities. Based on several considerations, we choose the frequency band between $16 \mathrm{kHz}$ and $20 \mathrm{kHz}$ for the signal.

4. Due to outliers in acoustic ranging, we may miss some pairwise distances with a small probability. The small search scopes set in step 4 of the algorithm help ensure the rigidity. 


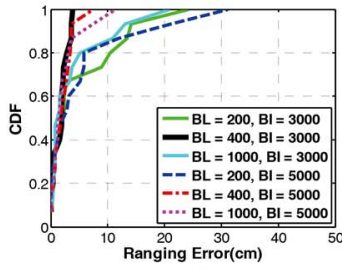

(a)

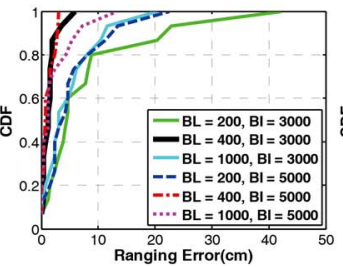

(b)

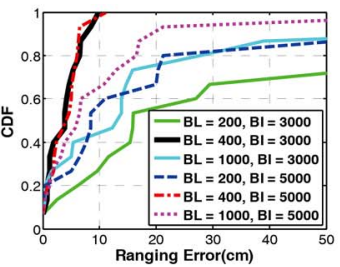

(c)

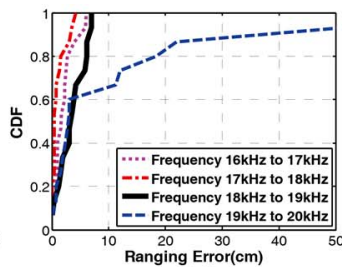

(d)

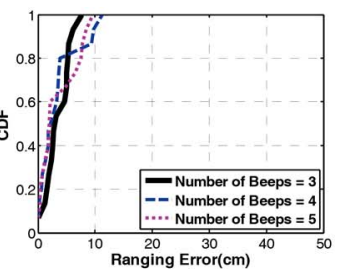

(e)

Fig. 6. Beep design: results obtained from HTC EVO. (a) BL and $\mathrm{BI}$ design, $100 \mathrm{~cm}$. (b) $\mathrm{BL}$ and $\mathrm{BI}$ design, $200 \mathrm{~cm}$. (c) $\mathrm{BL}$ and $\mathrm{BI}$ design, $300 \mathrm{~cm}$. (d) Frequency design, $300 \mathrm{~cm}$. (e) Number of beeps design, $300 \mathrm{~cm}$.

The majority of the background noise are located at the lower frequency band (e.g., conversation between $300 \mathrm{~Hz}$ to $3400 \mathrm{~Hz}$, music from $50 \mathrm{~Hz}$ to $15 \mathrm{kHz}$, which covers almost all naturally occurring sounds). $16-20 \mathrm{kHz}$ is still audible to human ears [15], but much less noticeable and thus present less disturbances. We also found that the current cell phone microphones are more sensitive to high frequency sounds than human ears [37]. A high frequency beep at the edge of the microphone's frequency response curve make it both easier to filter out noise and renders the signal un-noticeable to most people.

Our sound signal consists of several evenly paced beeps of equal lengths. We study how to set the number, length and frequency of the beeps, and the length of the intervals in Section 4.2.1. They all impact ranging accuracy and time. For example, too short a beep may not be picked up by the microphone, while too long a beep will add more delay to the ranging.

\subsubsection{Beep Detection}

Beep detection determines exactly when the beep signal arrives and it is critical to the accuracy of relative ranging. We investigate the behaviors of two most common signal detection methods, change-point detection and correlation-based under high frequency band.

Change-Point Detection Method. This method requires the beep have distinct energy uniformly distributed over a short frequency band (e.g., 16-18kHz). Given that band, this method first filters out the background noise using a Short Time Fourier Transform (SIFT). It then identifies the first strong signal that deviates from the noise in the targeted frequency band. The observed energy distributions of the recorded signal differ significantly before and after the arrival of the beep signal. Sequential change-point detection technique is adopted to identify the arrival of the signal, i.e., the exact time point of changed distribution [37].

Correlation-based Method. This method uses a chirp signal of a much shorter length. It correlates the emitted chirp sound with the recorded signal using L2-norm crosscorrelation, and picks as the signal detection time when the correlation value reaches the maximum. To be robust to multipath, the earliest sharp peak in the correlation values is used as the signal detection time [29].

\subsection{Detailed Design}

Through extensive experiments we find that change point has much higher accuracy than correlation. We will present the signal design for change point first, then compare the two methods under different environments.

\subsubsection{Sound Signal Parameters}

Experimental Setup. We used HTC EVO and Android Developer Phone 2 (ADP2) phones and $44.1 \mathrm{kHz}$ sampling rate in recording. During each test, two smartphones of the same model are used. One phone emits the sound first. Upon receiving the signal, the other phone emits the sound and both phones record. The relative ranging is computed by obtaining the time difference based on the signal detection in the recorded sounds by the two phones as described in Section 4.1.1. We spaced the two phones apart at $100 \mathrm{~cm}$, $200 \mathrm{~cm}$, and $300 \mathrm{~cm}$ respectively. Each curve in our results is obtained with 30 runs.

Beep Length (BL) and Beep Interval (BI) analysis. Fig. 6(a)-(c) presents the cumulative distribution function (CDF) of the ranging error when the beep length is set to 200, 400 and 1000 samples and the beep interval is set to 3000 and 5000 samples, respectively. The frequency band is $18 \mathrm{kHz}$ to $19 \mathrm{kHz}$. It is clear that beep length of $400 \mathrm{sam}-$ ples achieves the best performance: the ranging error is around $10 \mathrm{~cm}$ even when the two phones are placed $300 \mathrm{~cm}$ away, and the error is similar with beep interval of 3000 and 5000 samples. We thus choose beep length of 400 samples and beep interval of 3000 samples in our system implementation.

Beep Frequency Band. With the above settings, we examine the performance of different frequency bands beyond 15kHz. Fig. 6(d) shows the results when varying the frequency band from $16 \mathrm{kHz}$ to $20 \mathrm{kHz}$ with two HTC EVO phones $300 \mathrm{~cm}$ apart (better results are obtained in $100 \mathrm{~cm}$ and $200 \mathrm{~cm}$ cases). The ranging errors are less than $10 \mathrm{~cm}$ when the beep frequency is below $19 \mathrm{kHz}$. Similar experiments are conducted for ADP2 and we find that the highest frequency band producing comparable ranging results is $16-17 \mathrm{kHz}$. We choose $16-17 \mathrm{kHz}$ range for ADP2 and $18-19 \mathrm{kHz}$ for $\mathrm{HTC}$ EVO in our system.

Number of Beeps (NB). Outliers can occur in ranging results due to dynamic factors in the environment. The sound signal should contain multiple beeps, so as to obtain multiple ranging estimates and filter out outliers. However, too many beep in the sound signal may make it more susceptible to multi-path distortions. Fig. 6(e) shows the ranging error when different numbers of beeps are employed under frequency band 18$19 \mathrm{kHz}$ with $\mathrm{BL}=400$ samples and $\mathrm{BI}=3000$ samples. Basically, comparable performance (less than $10 \mathrm{~cm}$ ) is observed across three to five beeps. In our implementation, we used three beeps, which is robust up to two outliers on opposite side of the true distance. We observe 


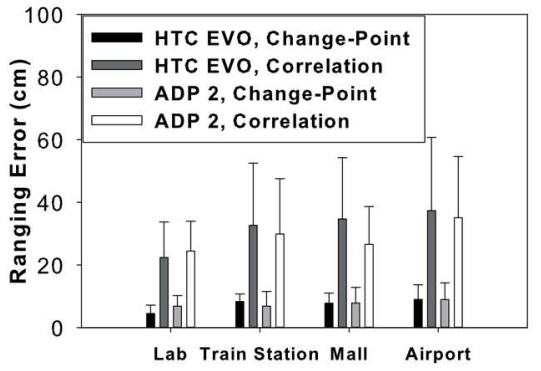

(a)

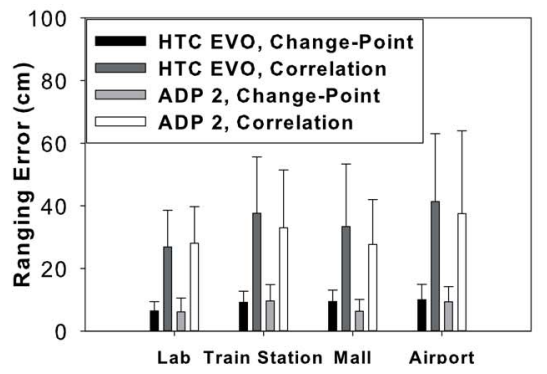

(b)

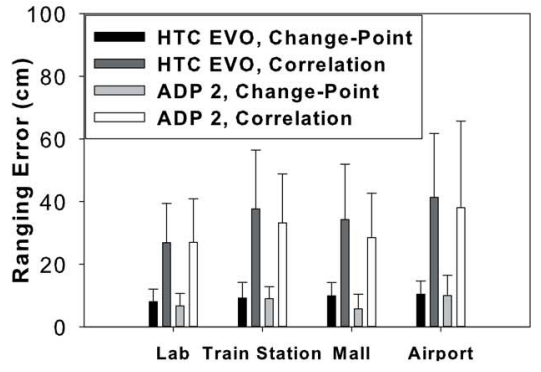

(c)

Fig. 7. Ranging errors under different environments. (a) $100 \mathrm{~cm}$. (b) $200 \mathrm{~cm}$. (c) $300 \mathrm{~cm}$.

that the probability of getting reliable results is more than $95 \%$.

\subsubsection{Robustness to Various Noises}

To evaluate the robustness of the design, we conduct a comprehensive study under various environments including lab, shopping mall, train station, and airport. There are all kinds of noises during our test such as human talking, radio broadcasting, dog barking, and trolley rolling. In each scenario, two phones are placed $100 \mathrm{~cm}, 200 \mathrm{~cm}$, and $300 \mathrm{~cm}$ apart.

Fig. 7 shows the median and 90th percentile ranging error using change-point detection method and correlationbased method respectively under high frequency band. For change-point, we use $16-17 \mathrm{kHz}$ for ADP2 and $18-19 \mathrm{kHz}$ for HTC EVO. The sound signal follows the previous parameters in Section 4.2.1. For correlation method, we use $16 \mathrm{kHz}-20 \mathrm{kHz}$ for both phone types and the chirp signal length is $50 \mathrm{~ms}$ [29]. We find that change-point detection significantly outperforms correlation in all cases: it has consistent low median errors around $10 \mathrm{~cm}$, while that of correlation ranges over $20 \mathrm{~cm}$ to $40 \mathrm{~cm}$; its 90 th percentile error is always below $20 \mathrm{~cm}$, while that of correlation can go up to $65 \mathrm{~cm}$. We thus choose change-point detection method for acoustic ranging in our system.

\subsection{Server Based Emission Schedule}

Identify Nearby Peers. A target phone needs to find which peers are nearby to get their help. Since large errors may exist in the WiFi location estimates for the target and peers, using WiFi localization alone is not reliable. We let the target phone emit a customized sound signal containing a single beep to identify truly nearby peers. Only phones really close enough can detect the signal. Those willing to help can send their IDs to the server. The server will then instruct these peers when they should emit the ranging signal.

Beep Emission Strategy. To speed up the ranging process, multiple phones need to emit sounds in a short time. Due to the lack of synchronized clocks, this may lead to interference among beeps from different phones, thus prohibiting accurate signal detection. There are two options to avoid such interference: time-division multiplexing or frequency-division multiplexing. We have tested concurrent emission of signals of different frequency bands, but find that interference still exists due to energy leakage among nearby frequency bands.
We employ time-division multiplexing based on server scheduling. ${ }^{5}$ We divide the time into slots of length $t_{s}$ long enough for the beep emission of one phone (e.g., 14000 samples or about $0.3 \mathrm{~s}$, for the previous signal parameters and a beginning padding of 3000 samples). After receiving the IDs of all phones, the server comes up with a back-to-back schedule for them. It sends the schedule to them, with the $i$ th phone starting beeping after a delay of $T_{p}+i \times t_{s} . T_{p}$ is some extra buffer to accommodate small variations in the reception of the schedule at different phones.

Each phone records beeps from others when its time slot has not come and plays the sound during its time slot. After finishing the whole schedule, all phones send their IDs and recorded files to the server, which performs signal detection and estimates distances.

\subsection{Virtual Synchronization}

The beep emission strategy requires peer phones synchronize their clocks due to the employment of time-division multiplexing. One natural choice is to have the server send an synchronization message to all the smartphones. We find, however, the round trip time (RTT) delay between smartphones and the server varies significantly, ranging from $2 \mathrm{~ms}$ to $149 \mathrm{~ms}$, when the server is connected with AP via Ethernet. The large variation of the RTT delay makes this method unreliable; different phones are not able to align their time slots well and collisions are inevitable.

To solve this problem, we leverage the recruiting signal, which is originally used to discover nearby phones for virtual synchronization among the involved smartphones. That is, each smartphone uses the arrival time of the recruiting signal as the common reference time point for beep emission schedules. The synchronization error thus comes from the different beep propagation latencies from the target phone to different peer phones (which is usually in a few milliseconds), and the difference in detecting the recruiting signal in peers phones (which is also a few milliseconds [29]. When extra buffer time $T_{p}$ much larger than the errors is "padded" to beeping time slots, such small synchronization errors will not cause collisions of acoustic signals.

Fig. 8 illustrates the process of virtual synchronization using the recruiting signal. The target phone first emits recruiting signal to recruit nearby peers. These recruited

5. We investigate an alternative where each phone randomly choose a timeslot to emit sound, but find that much more time slots are needed and it is difficult to distinguish the signals from different phones. 


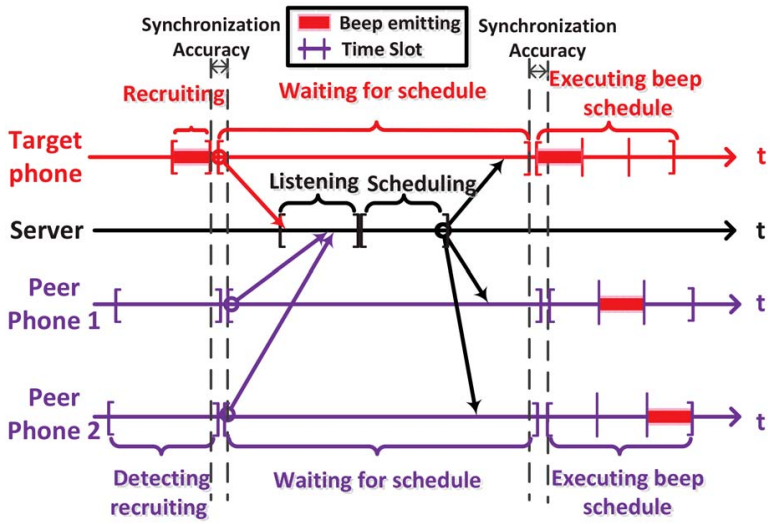

Fig. 8. Illustration of synchronization using recruiting signal.

peers will use the detected arrival time of the recruiting signal as a common reference time point. Each of the phones will wait for the same amount of fixed delay before executing the beep emission schedule. This ensures that the starting times of the schedule at different phones have the same synchronization error as the detections of the recruiting signal. The waiting time is long enough to ensure that during this period each of the phones has sent its ID to the server, the server has come up with the schedule and sent the schedule to each of the phones.

In our prototype, the recruiting signal is designed at the frequency band between $16 \mathrm{kHz}$ and $17 \mathrm{kHz}$, where both ADP2 and HTC EVO phones can reliably detect it. Different from the signal used for acoustic ranging, the recruiting signal is a single beep with the length of 800 samples. The enlarged duration of recruiting signal enables the phones to identify the presence of recruiting signal more reliably. In our prototype we find such a virtual synchronization method avoids collisions of beeping signals.

\section{ImPLEMENTATION AND EVALUATION}

In this section, we first describe how to detect the presence of large errors in the initial WiFi localization results in Section 5.1. We present the experimental methodology, testing scenarios and evaluation metrics in Section 5.2. We implement a prototype of our proposed system and report its performance in Section 5.3, including the accuracy, localization latency, and incurred energy consumption using Android phones. To capture the statistical performance of our scheme, we apply a trace-driven approach to conduct an extensive study of the impact of the quality and quantity of peers in Section 5.4. Finally, we study the sensitivity of our algorithm to orientation estimation in Section 5.5 and show the algorithm's performance under different realworld environments, such as train station, shopping mall and airport, in Section 5.6.

\subsection{Detecting the Presence of Large Errors}

The system needs to detect the presence of large errors in the initial WiFi position estimation for two purposes. First, target devices need this to help decide whether they should trigger peer assistance. Second, when selecting peers, we find that only those with small initial errors can serve as reliable reference points. However, the system does not
TABLE 2

Detection Results of Large Errors

\begin{tabular}{|l|c|c|c|c|}
\hline Localization Error (meter) & Mean & Median & $\mathbf{9 0 \%}$ & Maximum \\
\hline \hline Peer assisted (3 peers) & 1.7 & 1.4 & 2.6 & 3.1 \\
\hline Peer assisted (2 peers) & 2.0 & 1.7 & 3.2 & 4.5 \\
\hline \hline Peer assisted (3 peers, two modals) & 1.8 & 1.6 & 2.9 & 3.7 \\
\hline Peer assisted (2 peers, two modals) & 2.2 & 2.3 & 3.6 & 4.8 \\
\hline \hline WiFi & 3.8 & 3.2 & 5.9 & 6.8 \\
\hline
\end{tabular}

know the true location, how can it tell whether large errors exist in a position estimation? Based on the insights on large errors described in Section 2, we use 3 simple rules for reliable detection.

Rule I. Examine the probability of large errors at the initial estimated location. ${ }^{6}$ If a location has a large probability (e.g. $>0.8$ ) for an error beyond a threshold (e.g., $4.5 \mathrm{~m})$, declare the presence. This rule deals with consistent large errors at specific locations caused by permanent environmental settings (i.e., Case 1 in Section 2.3). For each location in the training data, we randomly pick 5 from the 60 samples to compute a location estimate, and repeat 400 times to compute the probability of large error occurrence.

Rule II. Examine the two sets of APs detected in the samples and in the training data at the estimated location. If the ratio of the number of common APs to the number of all detected APs is below a certain threshold, declare the presence.

Rule III. Compare the standard deviations of the RSS measurements on each AP in the samples and the training data at the initial estimated position. Among all common APs, if the fraction of APs having larger standard deviations in the samples exceeds a threshold, declare the presence.

Rules II and III deal with dynamic changes caused by transient factors (i.e., Case 2 in Section 2.3). They measure the statistical difference between the samples and the training data at the estimated location.

Intuitively, the system should be aggressive in declaring the presence for large errors, i.e., upon the detection from any of the 3 rules. Thus most targets having large errors are detected, and a peer with slight suspicion of having large errors is excluded. The cost is a few targets with smaller errors unnecessarily triggering peer assistance (but further improving accuracy), or peers with small errors excluded from assistance.

The threshold values for the three rules are derived based on the statistical hypothesis test and set such that they minimize false classification on the training data. The detection results are shown in Table 2. For example, in the office environment, we can use $4.5 \mathrm{~m}, 82 \%, 62 \%$ for detecting bad targets; $1.5 \mathrm{~m}, 42 \%$ and $90 \%$ for excluding bad peers. We find that collectively, $87 \%$ of bad targets are correctly identified, and among peers selected, 90\% indeed have errors less than $1.5 \mathrm{~m}$. Similarly, in the gym environment, we observe that $89 \%$ of bad targets are correctly identified with error larger than $4.5 \mathrm{~m}$, and $88 \%$ of the peers selected have errors less than $1.5 \mathrm{~m}$.

6. The nearest location in the interpolated training data set is actually used. Similar for the other two rules. 


\subsection{Evaluation Methodology}

We conduct experiments using Android Developer Phone 2 and HTC EVO. Both phones support $44.1 \mathrm{kHz}$ audio sampling. HTC EVO uses $512 \mathrm{MB}$ RAM and $1 \mathrm{GHz}$ Qualcomm QSD8650 processor, while ADP2 192 MB RAM and slower 528MHz MSM7200A processor. ${ }^{7}$

We generate the sound file based on the design in Section 4.1.2, which consists of three beeps, each created by uniformly distributing white noise and then bandpass filtered to $16-17 \mathrm{kHz}$ for ADP2 and $18-19 \mathrm{kHz}$ for HTC EVO.

We implement a system prototype including an Android smartphone app and a backend server, both written in Java. The server runs on Lenovo Thinkpad X201 with Intel Core i5 2.53GHz processor and 4GB DDR3 RAM. The app does WiFi RSS sampling, acoustic emitting and recording. The measurements are sent to the server for processing. The app has been tested for both ADP2 and HTC EVO. Both the server and app are multi-threaded so that they can perform multiple tasks in parallel to speed up the whole process.

Experimental Scenarios. We use the prototype to validate our algorithm design and measure practical performance. In particular, we select 10 target phone locations with large errors, each with different combinations of peer locations from the office setup in Fig. 1. Then we repeat the peer assisted localization for each target/peer location combination 10 times to measure the localization accuracy, total latency and energy consumption. In total we have 100 combinations of target/peer locations for the prototype experiments.

Trace-driven Statistical Performance. To capture the overall statistical performance under factors that we do not have enough manpower/device (e.g., more numbers of peers) or control (e.g., noise in different environments), we conduct trace-driven experiments. We use 200 combinations of target/peer locations in our office setup, feed the training data as WiFi samples, and distances perturbed with errors following the same distribution as results obtained in real environments (e.g., train station, shopping mall, and airport), as input to the peer-assisted algorithm. We study its the effectiveness of peer selection rules (Section 5.1), and the impact of various factors such as the number of peers, quality vs. quantity of peers, orientation estimations.

Metrics. We use the localization error to quantify the accuracy. We measure the total time needed to complete the peer assistance process, and decompose the total time to understand the dominant factors. We also measure the energy consumption using tools in [39] that is shown to achieve accuracy within $5 \%$.

\subsection{Prototype Performance}

Localization Accuracy. We evaluate the accuracy in office environment for 3 and 4 phones ( 2 and 3 peers) due to limited manpower and device availability. Peers have WiFi localization errors under $1.5 \mathrm{~m}$, and error of the target is over $3 \mathrm{~m}$. As shown in Table 3, we observe that the peer assisted method can reduce the maximum error from $6.8 \mathrm{~m}$ to $4.5 \mathrm{~m}$ and $3.1 \mathrm{~m}$ with 2 peers and 3 peers respectively. For median error, there is at least $1.5 \mathrm{~m}$ accuracy improvement.

7. We encountered some problem doing concurrent sound playing and recording on iPhone and are still investigating the issue.
TABLE 3

Localization Accuracy of the Prototype: 2 and 3 Peers for a

Target Phone with Large Initial Errors (> $3 \mathrm{~m}$ )

\begin{tabular}{|l|c|c|c|c|}
\hline Localization Error (meter) & Mean & Median & $\mathbf{9 0 \%}$ & Maximum \\
\hline \hline Peer assisted (3 peers) & 1.7 & 1.4 & 2.6 & 3.1 \\
\hline Peer assisted (2 peers) & 2.0 & 1.7 & 3.2 & 4.5 \\
\hline \hline Peer assisted (3 peers, two modals) & 1.8 & 1.6 & 2.9 & 3.7 \\
\hline Peer assisted (2 peers, two modals) & 2.2 & 2.3 & 3.6 & 4.8 \\
\hline \hline WiFi & 3.8 & 3.2 & 5.9 & 6.8 \\
\hline
\end{tabular}

Furthermore, more peers benefit the localization performance: the maximum error for 3 peers further reduces $1.4 \mathrm{~m}$ compared with 2 peers. We also test our system when there are different smartphone models involved in localization process. We find our system achieves comparable localization performance with a small variation on maximum and median error $(\sim 0.3 m)$.

Overall Latency and Decomposition. We report the latency measurements of major components in the peer assistance process. The recruiting takes about $0.5 \mathrm{~s}$, which is independent of the number of peers involved. Then the scanning of $5 \mathrm{WiFi}$ samples takes about $4.8 \mathrm{~s}$ on phones (HTC EVO), while in parallel the beep emitting takes $1.5 \mathrm{~s}$ (4 phones, 0.3s time slot, 1 extra buffer slot), the file uploading $1 \mathrm{~s}$, the TOA on server 1.2s. After WiFi and TOA results, the algorithm takes another $0.7 \mathrm{~s}$ on server. In total that is 6.0 s (i.e., $0.5+\max (4.8,3.7)+0.7)$. Since most other components can finish in parallel and faster than WiFi scan, our system does not pose much more latency than required in the original WiFi localization.

The algorithm execution time in peer assisted localization is dominated by the computation of rigid graph mapping, which involves a number of matrix operations. The JAVA language itself however does not provide any optimization for linear algebra. Fortunately, there are some ongoing projects on building libraries for high-performance linear algebra related computation in Java, e.g., JAMA [22], Parallel Colt (PColt) [34], and JBLAS [6]. JAMA is a basic linear algebra library for Java providing standard matrix class. PColt is a multithreaded version of Colt [23] library that can provide high performance scientific computing in Java. JBLAS is a fast linear algebra library implementation rooted in native BLAS [24] library.

We have implemented rigid graph mapping in Java with the above three libraries. We observe that the implementation with JBLAS has the shortest algorithm execution time, thanks to its multithreaded implementation. We thus finalize our implementation with JBLAS. In addition, we assign independent threads for every translational movement in the rigid graph mapping to further shorten the algorithm execution time. Essentially, our Java implementation of the rigid graph mapping finishes in 2.53s on average, which is less than the algorithm execution time optimized with Matlab implementation. When powerful hardware is available (e.g., a Mac with a 16 -core i7 CPU running at $2.9 \mathrm{GHz}$ and 16GB RAM), the algorithm execution time can be as low as $0.72 \mathrm{~s}$ at half resource utilization.

Energy Consumption. We also estimate the energy overhead using tools and methodology in [39]. The WiFi scan, sound emitting/recording and file uploading are the three major components. WiFi scan of $4.8 \mathrm{~s}$ takes about 0.12 Joule. 


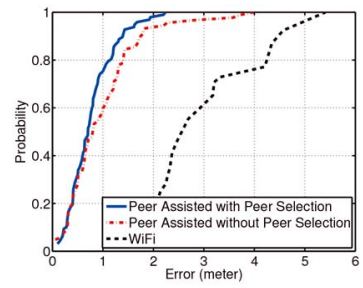

(a)

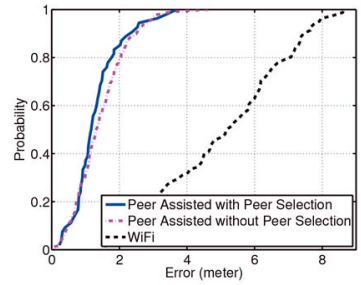

(c)

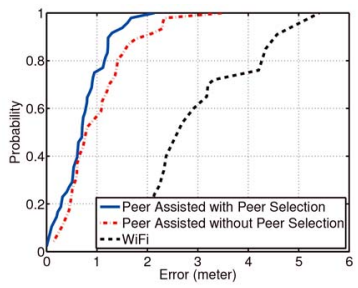

(b)

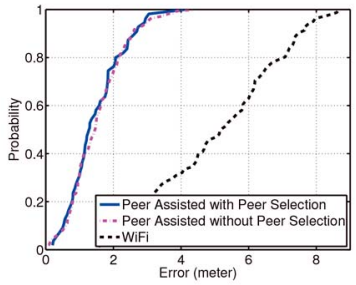

(d)

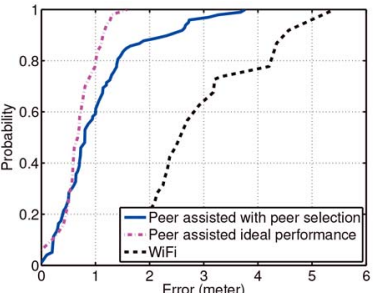

(a)

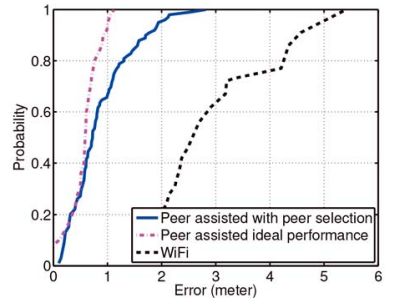

(c)

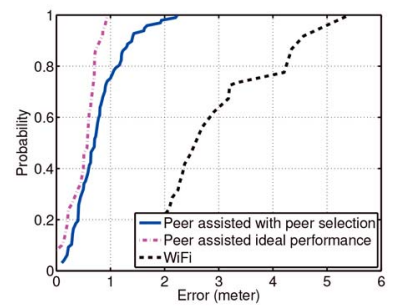

(e)

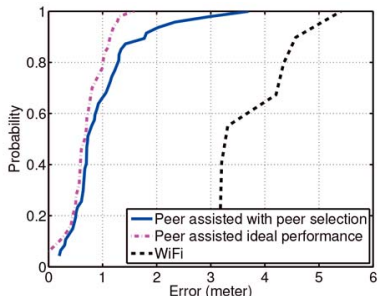

(b)

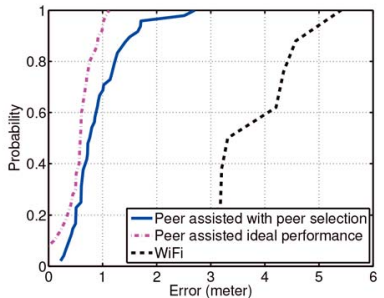

(d)

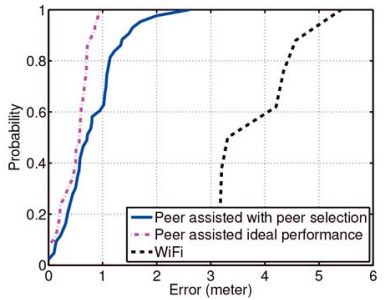

(f) File uploading is about 2.1 Joules. In total it is 2.57 Joules, with file uploading dominating at $80 \%$. All these happen in about $8 \mathrm{~s}$, translating into about $320 \mathrm{~mW}$ additional power consumption. This is smaller than the average power of a phone (e.g., HTC Evo lasts 12.7 hours with average power of $450 \mathrm{~mW})$. We believe such overhead does not pose a burden for the battery life. Furthermore, the peer assisted localization is triggered only when the user requests to improve the localization accuracy or the user is willing to help upon the request from others. Thus the increased power consumption occurs only during the short periods of peer assistance; it does not pose a continuous burden for phones.

\subsection{Overall Statistical Performance}

We next use trace-driven experiments by feeding the results of initial WiFi estimates and error-perturbed acoustic ranging results as inputs to capture the statistical behavior of our peer-assisted method.

Effect of Peer Selection. We first analyze the effect of the 3 peer selection rules (Section 5.1) on localization accuracy. Fig. 9(a) and (b) shows the localization error CDFs of those testing points with initial WiFi localization errors exceeding $1.5 \mathrm{~m}$ for both HTC EVO and ADP2 in the office environment. We observe great performance improvements with and without peer selection with CDF curves shifting to the left significantly for both phones. In particular, the median error exhibits a 75\% improvement under peer assistance with and without peer selection. We find that peer selection is essential for improving localization accuracy of points with large errors: the long CDF tail has been reduced $60 \%$ from $5.5 \mathrm{~m}$ to $2.2 \mathrm{~m}$. This shows that peers filtered by the rules are more reliable reference points and constitute more accurate constraints on the target location.

Impact of Environment. The localization results in the gym environment are shown in Fig. 9(c) and (d). We find
Fig. 10. Peer-assisted localization performance for target phone under different number of peers for HTC EVO in office environment. The first colomn is results when the target WiFi error is greater than $1.5 \mathrm{~m}$ and the second column with target WiFi error greater than $3 \mathrm{~m}$. (a) 3 peers (target WiFi error $>1.5 \mathrm{~m}$ ). (b) 4 peers (target WiFi error $>1.5 \mathrm{~m}$ ). (c) 5 peers (target WiFi error $>1.5 \mathrm{~m}$ ). (d) 3 peers (target WiFi error $>3 \mathrm{~m}$ ). (e) 4 peers (target WiFi error $>3 \mathrm{~m}$ ). (f) 5 peers (target WiFi error $>3 \mathrm{~m}$ ).

that the peer-assisted approach can help to reduce the large error from 8.8 meters to 4.2 meters. Such an improvement ( $\sim 52 \%)$ is comparable to that of $(\sim 60 \%)$ in the office environment. Moreover, under peer selection, the maximum error is further reduced by around 1 meter and 0.5 meters for HTC and ADP2 phones respectively comparing to the case without peer selection in the gym environment. The results show that our peer assisted approach can significantly improve the localization performance and the peer selection method can help to further reduce large errors. For the rest of the paper, we only present results with peer selection.

Impact of the Number of Peers. Fig. 10 shows the localization accuracy for the target phone when varying the number of peers at 3,4 and 5. In our experiments, the median error of initial WiFi localization is $1.5 \mathrm{~m}$, which indicates that about $50 \%$ of the testing target points have small errors below $1.5 \mathrm{~m}$ which are acceptable in most applications, while the other $50 \%$ of the target phones experiences errors beyond $1.5 \mathrm{~m}$. Based on this observation, we perform two sets of tests to evaluate the effectiveness of our peer-assisted approach: the first test applies to the $50 \%$ of the target phones with errors beyond $1.5 \mathrm{~m}$ as shown in the first column of Fig. 10, and the second test applies to those suffering from large errors exceeding $3 \mathrm{~m}$ (about $30 \%$ of target phones) as shown in the second column of Fig. 10. 


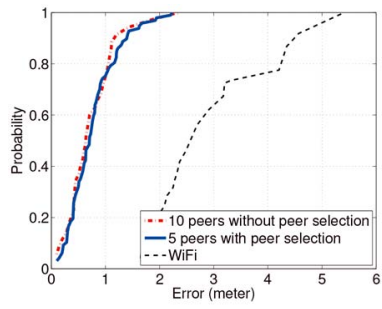

(a)

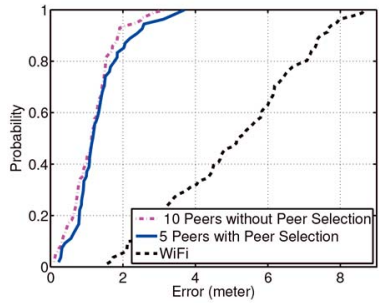

(c)

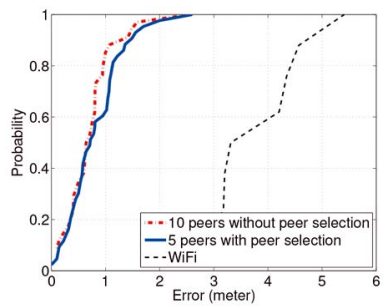

(b)

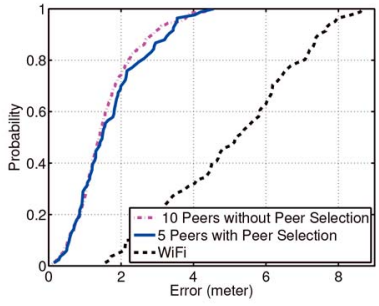

(d)
Fig. 11. Impact of peer quality vs. peer quantity in both lab and gym environment. (a) Target WiFi error $>1.5 \mathrm{~m}$ (Lab). (b) Target WiFi error $>3 \mathrm{~m}$ (Lab). (c) Target WiFi error $>1.5 \mathrm{~m}$ (Gym). (d) Target WiFi error $>3 m$ (Gym).

The most encouraging result is that our approach show consistently great performance improvement for both test cases, suggesting that our algorithm is highly effective to reduce large errors. In general, more peers lead to more improvements in accuracy: When the number increases from 3 to 5 in Fig. 10, the maximum error in the peerassisted curve reduces from about $3.8 \mathrm{~m}$ to $2.4 \mathrm{~m}$ for both test cases, while the median error stays more or less the same around $0.7 \mathrm{~m}$. This is because more peers pose more constraints and less uncertainty on the location of the target.

We also show the ideal performance where the true graph orientation and distances between phones are known for the algorithm. The maximum and median error are $1.5 \mathrm{~m}$ and $0.5 \mathrm{~m}$. For about $80 \%$ of the case, the accuracy is very close (within $0.4 \mathrm{~m}$ ) to the ideal performance. This indicates that our orientation angle estimation is quite accurate.

Quality vs. Quantity of Peers. One interesting question is how important is the quality versus the quantity of peers. Fig. 11(a) and (b) presents the localization error CDF when using peer assisted localization with 10 peers without selection and 5 peers with selection (based on the rules designed in Section 5.1) respectively. We find that the performance of using 5 peers with selection is comparable to that of using 10 peers without selection when applying to scenarios of target phones with WiFi error greater than $1.5 \mathrm{~m}$, and only slightly worse (only for the bottom $40 \%$ cases and differ by about $0.2 \mathrm{~m}$ ) when the target $\mathrm{WiFi}$ error is greater than $3 \mathrm{~m}$. We have similar observation in the gym environment as shown in Fig. 11(c) and (d). The performance of using 5 peers with peer selection is similar to that of using 10 peers without peer selection. Thus, by utilizing peer selection only half of the peers is needed to achieve a similar performance as when adopting peers blindly. This observation strongly suggests that peer quality is more important than peer quantity and peer selection is critical.

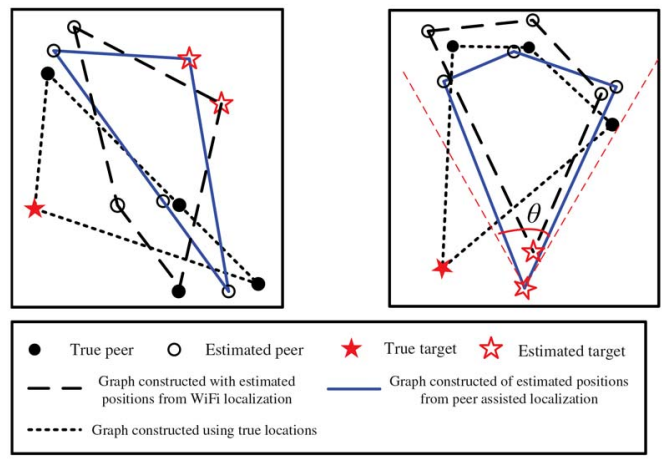

(a)

(b)

Fig. 12. Illustration of larger errors for peer assisted localization method. (a) Mirroring outliers. (b) Rotation outliers.

\subsubsection{Insights on Remaining Errors}

During our initial experiments we analyzed what happened for the points towards the tail of the CDF curve under peer assistance. We found two reasons limiting the accuracy improvement. The first is called "mirroring" (shown in Fig. 12(a)). When the peers are almost aligned on a straight line, the constructed rigid graph could be a flipped "mirror" of the ground truth. If the mirrored graph happens to have a smaller RSS distance summation, the target would be located to the opposite side of the line. By comparing the ranging measurements, we can tell whether such alignment happens and avoid using such peer combinations. We have addressed this problem in all previous results.

The second is due to errors in orientation when the target is relatively faraway from peers (shown in Fig. 12(b)). When the peers are close to each other, a small orientation deviation can move the target far away from its true location while the peers are still estimated close to their true locations. To reduce such error caused by the clustering effect of faraway peers, we identify the formed cluster by examining the angles formed by the estimated positions of target phone and any of two peers as shown in Fig. 12(b). We first draw lines from the estimated target to each estimated peer. We then obtain the maximum angle $\theta_{\max }$ among all the formed angles by any two of those lines. If the angle $\theta_{\max }$ is larger than a pre-defined threshold $\theta$, which serves to determine whether the estimated peers are close to each other, we will drop current peer selection and choose other peers around the estimated target. We have used $\theta=60^{\circ}$ in our implementation and applied such angle examination process when presenting the results in this section.

\subsection{Orientation Estimation and Sensitivity}

The peer-assisted localization accuracy is affected by the orientation estimation. we take a rigid graph of 4 phones constructed using their true distances to showcase the sensitivity of localization results to orientation errors. We rotate the graph so it deviates from its true orientation at a fixed degree, then we move the graph translationally to find the optimal locations. The results are shown in Fig. 13(a). We find that when the orientation deviation is larger than $24^{\circ}$, the average localization error becomes larger than that of initial WiFi localizations $(1.3 m$ in this case); it increases sharply to 8 meters when the orientation 


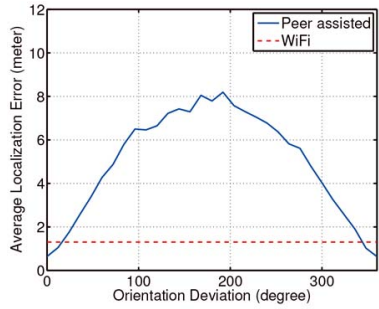

(a)

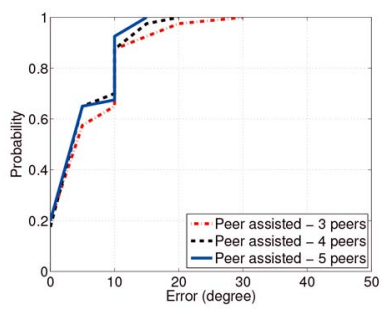

(b)

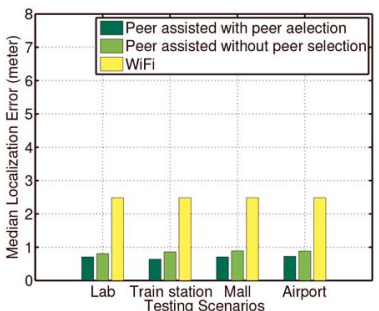

(a)

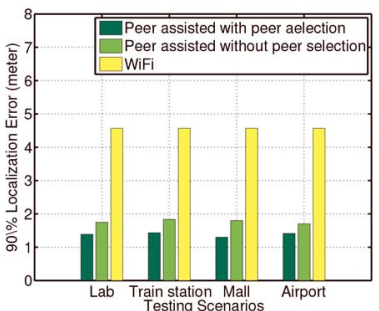

(b)
Fig. 13. (a) Average localization error under different orientation deviations. (b) Orientation estimation performance with different number of peers.

deviation approaches $180^{\circ}$. We examine cases with other numbers of peers, and find that generally the orientation deviation shall not exceed $20^{\circ}$, otherwise the improvement will be very limited. Fig. 13(b) shows the accuracy of orientation estimation under different number of peers. We find that our orientation estimation method is indeed very effective, which can restrict the error within $\pm 20^{\circ}$ and $\pm 10^{\circ}$ around the true angle for over $98 \%$ and $90 \%$ of the testing scenarios.

\subsection{Impact of Various Environments}

We further study the peer-assisted localization performance for the bottom $50 \%$ target points (i.e., those with $>1.5 \mathrm{~m}$ initial errors) with 5 peers, using ranging results perturbed with errors under various environments. In Fig. 14, we find that our localization performance only varies slightly when comparing real-world environmens (e.g., train station, shopping mall and airport) to the lab environment. The median errors are all around $0.7 \mathrm{~m}$ whereas the $90 \%$ errors are about $1.4 \mathrm{~m}$; even without peer selection, the median and $90 \%$ errors are around $0.8 \mathrm{~m}$ and $1.7 \mathrm{~m}$ respectively. Compared to the target's WiFi results of median error $2.5 \mathrm{~m}$ and $90 \%$ error $4.5 \mathrm{~m}$, our proposed method is quite robust to noises in different environments.

\section{Discussion}

Peer Involvement. Since peers need to spend energy and bandwidth for helping the target, we envision that whichever application that leverages our localization solution may have certain rewards (e.g., points, virtual currency) exchanged to compensate peers that help out. Such incentives are already present in many mobile applications. In our system, peers need to run a recruiting signal detection thread to know whether they are needed. To avoid running this thread continuously, the server may send push notifications to peers in a large area around a target upon its request for help. The notification triggers the thread; if no recruiting signal is detected after a timeout threshold, a peer will stop the thread. We note that the improvement in accuracy depends on the number and quality of peers. In general, more high quality peers lead to greater improvements. But even with just a few (e.g., 3) quality peers, there is still significant reduction in error.

Movements of Users. Peers might be moving during the acoustic ranging. Movements affect the accuracy only when they occur during the sound-emitting period, which happens concurrently with and takes less time than WiFi
Fig. 14. Localization performance under different environments with 5 peers. (a) Median Error. (b) 90-Percentile Error.

scanning (1.5s vs $4.8 \mathrm{~s}$ in our prototype). Thus we do not pose more constraints on movements than existing WiFi methods. If the peers are static or do not move too much in this 4.8 s interval, both the ranging and WiFi measurements are still accurate. In many public spaces it is common for many users to remain still for a short while (e.g., resting in airports, window-shopping in stores). Movements before and after the WiFi scanning (and beeping) do not affect the accuracy. We recognize that less peers may be suitable due to movements and plan to examine additional input such as acceleration to filter out those that have moved too much. The main latency bottleneck is the WiFi scanning time, which depends on both hardware and OS that we do not have control. Our experiments show that the acoustic ranging design is robust to noises under different environments. One issue we plan to further investigate is the effects of clothes and human body. When phones are placed in pockets, the clothes or human body may attenuate the sound signal.

Triggering Peer Assistance. Our work provides the technology for peer assistance. However, it is eventually up to the users to decide when they desire such help. The rules provide some hints to users about the likelihood of large errors to help them make the decision. Users may also set up certain policies about under what kinds of conditions assistance is needed. One that always requires help gets high accuracy, at the cost of paying more points or virtual currency to others. In our system, more than one target phone may request assistance at the same time and the acoustic signals may collide if these targets are close to each other. Since users do not need assistance continuously, they request help only once in a while, e.g., when wondering which hallway to take to a train platform. So the likelihood of two nearby users requesting help simultaneously is small. When this happens, the server can distinguish them and provide assistance one at a time. This may delay the localization to some targets, but it still provides accurate results.

Practical Usage. The above 3 items are all practical factors that are taken into consideration during real system deployment. In addition, both our trace-driven and smallscale prototype tests in gym and office environments show the feasibility of the peer assisted localization system. And the acoustic ranging techniques have also been validated in various scenarios including shopping mall, airport and train station. We are under the process of validating our system in various environments and will provide a practical end-to-end system in the future. Meanwhile, we are 
also studying the security and privacy issues involved in our system by identifying the unique security problems and comparing with existing techniques addressing security concerns in Wi-Fi localization.

\section{Related Work}

Smartphone indoor localization has attracted tremendous interests recently. Methods in prior work mainly focus on employing more sensing modalities, less infrastructure support, and reducing the efforts of building signal maps [2], [9], [10], [19]. SurroundSense [2] utilizes multiple sensing modalities (e.g., cameras, microphones and accelerometers) available on smartphones to perform logical localization (e.g., different stores) via ambiance fingerprinting by combining optical, acoustic, and motion attributes. WiGEM [17] proposes a learning based approach that uses the Gaussian Mixture Model (GMM) and employs Expectation Maximization (EM) to estimate the model parameters without relying on labor-intensive "training." It is robust to multiple factors (including device and power level variability, mobility, and changes of indoor spaces) that many training-based systems are susceptible to. WILL [9] aims to perform indoor logical localization without the need of building radio signature maps ahead of time. It exploits abrupt signal changes through walls and accelerometers to infer user movements and achieve room level accuracy. EZ [10] is another work that targets configuration-free indoor localization by utilizing geneticbased algorithms. Large errors still exist with 50 and 80 percentile errors at $7 \mathrm{~m}$ and $10 \mathrm{~m}$, respectively. WiGEM, WILL and EZ are complementary to our system for reducing the efforts of building the radio signal map during training.

Few studies have leveraged the unique peer constraints to assist smartphone localization. Virtual Compass [5] utilizes both WiFi and Bluetooth and results in a median error of $3-4 \mathrm{~m}$. Other studies require special hardware or infrastructure not readily available on smartphones [21], [27]. Centaur [28] combines the radio frequency and acoustic ranging measurements to improve the localization accuracy in an office environment. This work is primarily limited in an office environments due to the requirement of pre-deployment of several fixed anchor points. Our work leverages abundant peers in public spaces to reduce large errors. It aims at the most prevalent WiFi infrastructure and do not require any special hardware, which is essential for easy deployment.

There have been quite some work for acoustic positioning techniques such as Cricket [30], Bat system [20], ENSBox [16], and WALRUS [7]. Recently, several proposals have studied ranging between cell phones using low frequency bands (e.g., 2-6kHz). Beepbeep [29] proposes a ToA based acoustic-ranging method between two phones. It can achieve 1 or $2 \mathrm{~cm}$ accuracy in a range of $10 \mathrm{~m}$. Whistle [36] leverages multiple receivers with well-known locations to receive two sounds signals from the target. It avoids tight synchronization required in Time Difference of Arrival (TDoA) methods. Qiu et al. [32] utilizes the acoustic signal to estimate the relative position between two phones to support phone-to-phone games and apps.
Compared to the above work, we face a different challenge of fast and accurate ranging among multiple peer phones. We use server scheduling to coordinate multiple peer phones to avoid signal interference and collision for almost concurrent pairwise ranging. We propose signal design and detection methods that are light-weight in computation, robust to noisy environments, and much less perceptible to the human ear due to the adoption of high frequency band (e.g., 16-19kHz). Our system utilizes minimum auxiliary COTS sound hardware to reduce large errors incurred from general WiFi-based approaches.

For smartphone based localization using acoustic signals, Tarzia et al. [33] introduces a technique based on ambient sound fingerprint called Acoustic Background Spectrum. They exploit acoustic signals as fingerprints instead of measuring the ranging information between phones, and their localization granularity is at room level. Constandache et al. [12] deploys inaudible sound beacons randomly placed in the building, as a reference frame for correcting users' movement traces captured by the accelerometer and compass. However, this system requires an extra acoustic infrastructure and has localization errors around $8 \mathrm{~m}$ on average. In this paper, we exploit the constraints from nearby peer phones to achieve much higher localization accuracy, without the need for extra acoustic infrastructure.

\section{Conclusion}

Indoor localization on smartphones is critical to enable novel features for location based applications. However, existing approaches have yet to prove that they can satisfy what is desired in many business scenarios. Due to the prevalence of WiFi infrastructure, we set out to study the accuracy that WiFi localization can practically achieve on smartphones. We find that despite reasonable accuracies in many cases, the dynamic radio propagation poses fundamental limits and causes large errors. Inspired by the idea of relative positions of nearby peer devices as unique physical constraints on the possible location of a smartphone, we propose a peer assisted localization approach that leverages much more accurate distance estimate through acoustic ranging. A virtual synchronization strategy is proposed to avoid the possible sound signal collision among peer phones. Extensive experiments in both office and gym environments have demonstrated our approach successfully pushes further the limit of WiFi localization accuracy to what is empirically possible only under hundreds of APs, making WiFi a candidate for high accuracy localization. Our system does not pose much more latency than required in the original WiFi localization and has negligible impact on the battery lifetime.

\section{ACKNOWLEDGMENTS}

The preliminary results of this work have been published in ACM Mobicom 2012 [25]. This work is supported in part by the US National Science Foundation Grants CNS-0954020, CNS-1016303, CCF-1018270, CNS-1318751, CNS-1217387, and CNS-1318748. This work was done while H. Liu was at Stevens Institute of Technology. 


\section{REFERENCES}

[1] Shopkick Application [Online]. Available: http:/ / www.shopkick.com/

[2] M. Azizyan, I. Constandache, and R. R. Choudhury, "SurroundSense: Mobile phone localization via ambience fingerprinting," in Proc. ACM MobiCom, Beijing, China, 2009.

[3] P. Bahl, V. Padmanabhan, and A. Balachandran, "Enhancements to the RADAR user location and tracking system," Microsoft Res., Tech. Rep. MSR-TR-2000-12, 2000.

[4] P. Bahl and V. N. Padmanabhan, "RADAR: An in-building RF-based user location and tracking system," in Proc. IEEE INFOCOM, 2000.

[5] N. Banerjee et al., "Virtual compass: Relative positioning to sense mobile social interactions," in Proc. Pervasive, 2010.

[6] B. Blount and S. Chatterjee, "An evaluation of Java for numerical computing," in Computing in Object-Oriented Parallel Environments, Berlin, Germany: Springer 1998.

[7] G. Borriello, A. Liu, T. Offer, C. Palistrant, and R. Sharp, "WALRUS: Wireless acoustic location with room-level resolution using ultrasound," in Proc. ACM MobiSys, 2005.

[8] G. Chandrasekaran et al., "Empirical evaluation of the limits on localization using signal strength," in Proc. 6th Annu. IEEE Commun. Soc. Conf. SECON, Rome, Italy, 2009.

[9] W. Chenshu, Y. Zheng, L. Yunhao, and X. Wei, "WILL: Wireless indoor localization without site survey," in Proc. IEEE INFOCOM, Orlando, FL, USA, 2012.

[10] K. Chintalapudi, A. Padmanabha Iyer, and V. N. Padmanabhan, "Indoor localization without the pain," in Proc. ACM MobiCom, 2010.

[11] CNN. New Version of Google Maps Brings Indoor Floor Plans to Your Phone [Online]. Available: http://www.cnn.com/2011 11/30/tech/mobile/new-version-google-maps-indoors-wired/

[12] I. Constandache, X. Bao, M. Azizyan, and R. R. Choudhury, "Did you see bob?: Human localization using mobile phones," in Proc. ACM MobiCom, Chicago, IL, USA, 2010.

[13] B. J. Dil and P. J. M. Havinga, "RSS-based localization with different antenna orientations," in Proc. ATNAC, Auckland, New Zealand, 2010.

[14] R. Fan, Z. Tian, D. Linfu, J. Y. Li, and Q. Wan, "Indoor localization error measurements with multiple channels," in Proc. IEEE NSWCTC, Wuhan, Hubei, China, 2010.

[15] S. Gelfand and H. Levitt, Hearing: An Introduction to Psychological and Physiological Acoustics, New York, NY, USA: Marcel Dekker, 2004.

[16] L. Girod, M. Lukac, V. Trifa, and D. Estrin, "The design and implementation of a self-calibrating distributed acoustic sensing platform," in Proc. ACM SenSys, New York, NY, USA, 2006.

[17] A. Goswami, L. E. Ortiz, and S. R. Das, "WiGEM: A learningbased approach for indoor localization," in Proc. ACM CoNEXT, Tokyo, Japan, 2011.

[18] J. Graver, B. Servatius, and H. Servatius, Combinatorial Rigidity. Providence, RI, USA: American Mathematical Society, 1993.

[19] Y. Haibo et al., "FTrack: Infrastructure-free floor localization via mobile phone sensing," in Proc. IEEE PerCom, Lugano, Switzerland, 2012.

[20] A. Harter, A. Hopper, P. Steggles, A. Ward, and P. Webster, "The anatomy of a context-aware application," in Proc. ACM MobiCom, New York, NY, USA, 1999

[21] M. Hazas et al., "A relative positioning system for co-located mobile devices," in Proc. ACM MobiSys, New York, NY, USA, 2005.

[22] J. Hicklin et al. (2000). Jama: A Java Matrix Package [Online]. Available: http:/ / math.nist. gov/javanumerics/jama

[23] W. Hoschek. (2002). The Colt Distribution: Open Source Libraries for High Performance Scientific and Technical Computing in Java [Online]. Available: http://nicewww.cern.ch/hoschek/colt/index.htm

[24] C. Lawson, R. Hanson, D. Kincaid, and F. Krogh, "Basic linear algebra subprograms for fortran usage," ACM Trans. Math. Softw., vol. 5, no. 3, pp. 308-323, Sep. 1979.

[25] H. Liu et al., "Push the limit of WiFi based localization for smartphones," in Proc. ACM Mobicom, Istanbul, Turkey, 2012.

[26] Y. Liu, Q. Wang, J. Liu, and T. Wark, "MCMC-based indoor localization with a smart phone and sparse WiFi access points," in Proc. IEEE PERCOM Workshops, Lugano, Switzerland, 2012.
[27] M. Minami, Y. Fukuju, K. Hirasawa, and S. Yokoyama, "DOLPHIN: A practical approach for implementing a fully distributed indoor ultrasonic positioning system," in Proc. ACM UbiComp, Nottingham, U.K., 2004.

[28] R. Nandakumar, K. K. Chintalapudi, and V. N. Padmanabhan, "Centaur: Locating devices in an office environment," in Proc. ACM MobiCom, New York, NY, USA, 2012.

[29] C. Peng, G. Shen, Y. Zhang, Y. Li, and K. Tan, "BeepBeep: A high accuracy acoustic ranging system using cots mobile devices," in Proc. ACM SenSys, New York, NY, USA, 2007.

[30] N. Priyantha, A. Chakraborty, and H. Balakrishnan, "The cricket location-support system," in Proc. ACM MobiCom, New York, NY, USA, 2000

[31] A. Prorok, P. Tome, and M. Alcherio, "Accommodation of NLOS for ultra-wideband TDOA localization in single- and multi-robot systems," in Proc. IEEE IPIN, Guimaraes, Portugal, 2011.

[32] J. Qiu, D. Chu, X. Meng, and T. Moscibroda, "On the feasibility of real-time phone-to-phone 3D localization," in Proc. ACM SenSys, Washington, DC, USA, 2011.

[33] S. P. Tarzia, P. A. Dinda, R. P. Dick, and G. Memik, "Indoor localization without infrastructure using the acoustic background spectrum," in Proc. ACM MobiSys, New York, NY, USA, 2011.

[34] P. Wendykier and J. Nagy, "Parallel colt: A high-performance Java library for scientific computing and image processing," ACM Trans. Math. Softw., vol. 37 no. 3, Article 31, Sep. 2010.

[35] J. Xiong and K. Jamieson, "ArrayTrack: A fine-grained indoor location system," in Proc. USENIX NSDI, Berkeley, CA, USA, 2013.

[36] B. Xu, R. Yu, G. Sun, and Z. Yang, "Whistle: Synchronization-free TDOA for localization," in Proc. IEEE ICDCS, Minneapolis, MN, USA, 2011

[37] J. Yang et al., "Detecting driver phone use leveraging car speakers," in Proc. ACM MobiCom, Minneapolis, MN, USA, 2011.

[38] M. Youssef and A. Agrawala, "The horus WLAN location determination system," in Proc. ACM MobiSys, 2005.

[39] L. Zhang et al., "Accurate online power estimation and automatic battery behavior based power model generation for smartphones," in Proc. IEEE CODES/ISSS, Scottsdale, AZ, USA, 2010.

[40] Z. Zhang et al., "I am the antenna: Accurate outdoor AP location using smartphones," in Proc. ACM MobiCom, Las Vegas, NV, USA, 2011.

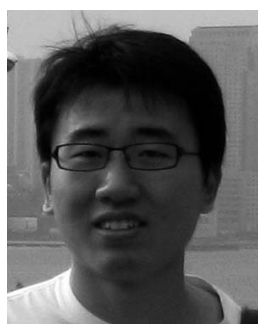

Hongbo Liu is an Assistant Professor with the Department of Computer Information and Graphics Technology at IUPUI. He received the Ph.D. degree in electrical engineering from Stevens Institute of Technology, Hoboken, NJ, USA. His current research interests include cyber security and privacy, mobile and pervasive computing, and smart grid. He is the recipient of the Best Paper Award from ACM MobiCom 2011 and the Best Paper Runner-up Award from IEEE CNS 2013. He is a member of the IEEE.

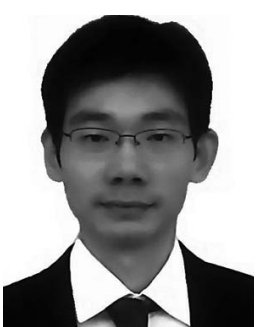

Jie Yang received the Ph.D. degree in computer engineering from Stevens Institute of Technology, Hoboken, NJ, USA, in 2011. He is currently an Assistant Professor with the Department of Computer Science at Florida State University, Tallahassee, FL, USA. His research interests include cyber security and privacy, and mobile and pervasive computing, with an emphasis on network security, smartphone security and applications, security in cognitive radio and smart grid, location systems and vehicular applications. His research is supported by National Science Foundation and Army Research Office. He is the recipient of the Best Paper Runner-Up Award from IEEE Conference on Communications and Network Security 2013 and the Best Paper Award from ACM MobiCom 2011. His research has received wide press coverage including MIT Technology Review, The Wall Street Journal, NPR, CNET News, and Yahoo News. He is a member of the IEEE. 


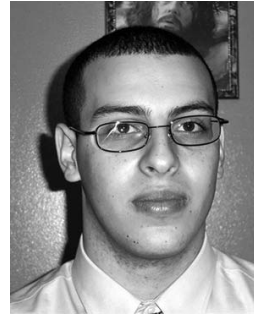

Simon Sidhom is currently a Senior Software Engineer in P97 Networks. He received the B.S. degree in cybersecurity as well as the M.S. degree in computer science both from the Stevens Institute of Technology, Hoboken, NJ USA. His graduate certifications include: system administration, cloud computing, serviceoriented computing, enterprise security and information assurance, and computer systems. His current research interests include acoustic localization, mobile systems, and computer security and privacy.

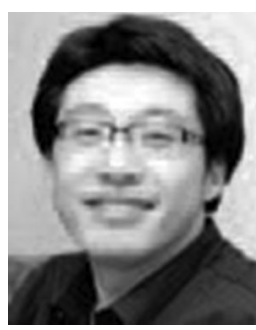

Yan Wang is a Ph.D. candidate with the Electrical and Computer Engineering Department at Stevens Institute of Technology, Hoboken, NJ, USA. His current research interests include mobile computing, information security, and privacy and pervasive computing. $\mathrm{He}$ is currently with the Data Analysis and Information SecuritY (DAISY) Lab with Prof. Yingying Chen. He is a student member of the IEEE.

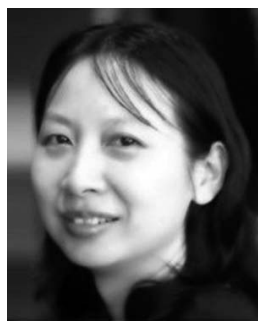

Yingying Chen is an Associate Professor with the Department of Electrical and Computer Engineering at Stevens Institute of Technology, Hoboken, NJ, USA. Her current research interests include cyber security and privacy, mobile computing, mobile healthcare, and wireless networks. She has published extensively in these areas in both journal articles and referred conference papers. She received the Ph.D. degree in computer science from Rutgers University, New Brunswick, NJ, USA. Prior to joining Stevens, she was with Alcatel-Lucent. She is the recipient of the US NSF CAREER Award and the Google Research Award. She also received NJ Inventors Hall of Fame Innovator Award. She is the recipient of the Best Paper Award from ACM International Conference on Mobile Computing and Networking (MobiCom) in 2011. She also received the IEEE Outstanding Contribution Award from IEEE New Jersey Coast Section each year from 2005 to 2009. Her research has been reported in numerous media outlets. She is a senior member of the IEEE.

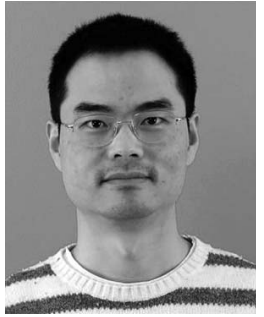

Fan Ye received the B.E., and M.S. degrees in automation and computer science in 1996 and 1999, both from Tsinghua University, Beijing, China. After receiving the Ph.D. degree in computer science from UCLA in 2004, he joined IBM T. J. Watson Research Center as a Research Staff Member. He is currently an Associate Professor with the Center for Energy-efficient Computing and Applications of EECS School, Peking University, Beijing, China. His current research interests include mobile computing and applications, mobile cloud, wireless and sensor networks.

$\triangleright$ For more information on this or any other computing topic, please visit our Digital Library at www.computer.org/publications/dlib. 